Ophthalmologe $2022 \cdot 119: 834-836$ https://doi.org/10.1007/s00347-021-01492-4 Eingegangen: 21. Juni 2021 Überarbeitet: 10. August 2021 Angenommen: 12. August 2021 Online publiziert: 15. September 2021 ๑ () Der/die Autor(en) 2021

\section{Venenastthrombose als frühe Manifestation von COVID-19}

\author{
Kristin Hösel (D) - Mark Saeger · Johann B. Roider \\ Universitätsklinikum Schleswig-Holstein - Campus Kiel, Kiel, Deutschland
}

\section{Anamnese}

Ein 55-jähriger Patient stellte sich mit seit circa 3 Tagen bestehender schmerzloser Visusminderung auf dem rechten Auge vor.

Zum Zeitpunkt der Diagnosestellung bestanden keine Grunderkrankung und keine Medikation.

\section{Befund}

Der bestkorrigierte Visus des betroffenen rechten Auges betrug 0,5. Der Augeninnendruck sowie vordere Augenabschnitt waren unauffällig. Fundoskopisch zeigten sich dilatierte Venen des temporalinferioren Gefäßbogens mit umgebenden Streifenblutungen sowie multiplen Cotton-Wool-Herden (- Abb. 1). Es bestand keine Glaskörperinfiltration. In der optischen Kohärenztomographie (OCT) zeigte sich ein zystoides Makulaödem. In der Fluoreszeinangiographie zeigte sich eine Venenastthrombose (VAT) des inferioren Gefäßbogens mit umgebender retinaler Ischämie sowie Schrankenstörung (• Abb. 2).

Der Visus des Partnerauges betrug 0,8. Inferior der Papille des linken Auges zeigte sich eine winzige retinale Hämorrhagie unterhalb der Papille bei sonst unauffälligem ophthalmologischem Status.

\section{Diagnose}

Der Patient wurde mit der Diagnose einer Venenastthrombose (Auge rechts) stationär aufgenommen.

\section{Therapie und Verlauf}

Am Tag der stationären Aufnahme wurde ein Nasen-Rachen-Abstrich auf SARS-CoV2-RNA untersucht (RT-PCR), welcher sich positiv zeigte. Auch auf Nachfrage verneinte der Patient Allgemeinsymptome, insbesondere Luftnot, Husten oder Geschmacksstörungen. Die Infektionskette war nicht bekannt. Die Körpertemperatur betrug $35,6^{\circ} \mathrm{C}$, der Blutdruck 151/98 $\mathrm{mm} \mathrm{Hg}$, die Herzfrequenz $82 / \mathrm{min}$ und die Sauerstoffsättigung $98 \%$. Die gängigen Entzündungsmarker waren im Normbereich (CRP $=2,5 \mathrm{mg} / \mathrm{l}, \mathrm{LDH}=177 \mathrm{U} / \mathrm{l}$, Troponin $\mathrm{T}=5,4 \mathrm{ng} / \mathrm{l})$.

Wir führten einen Bindehautabstrich (Schirmer-Streifentest) auf beiden Augen durch. In beiden Augen konnte SARS-CoV2-RNA nachgewiesen werden.

Der Patient wurde auf unsere Isolierstation verlegt und erhielt $100 \mathrm{mg}$ Prednisolon intravenös über 5 Tage mit folgender Entlassung in die häusliche Quarantäne.

Am fünften Tag nach Entlassung (etwa 14 Tage nach Auftreten der Sehstörung) wurde der Patient erneut stationär in der Klinik für Innere Medizin aufgenommen. Er berichtete nun über zunehmende Luftnot, Verschlechterung des Allgemeinbefindens, Fieber und Husten. Die Körpertemperatur betrug $38,4^{\circ} \mathrm{C}$, der Blutdruck $124 / 84 \mathrm{~mm} \mathrm{Hg}$, die Herzfrequenz 91/min und die Sauerstoffsättigung $92 \%$. Auch die Entzündungsparameter zeigten einen Anstieg: $\mathrm{CRP}=71,3 \mathrm{mg} / \mathrm{l}, \mathrm{LDH} 286 \mathrm{U} / \mathrm{l}$, Troponin $T=6,3 \mathrm{ng} / \mathrm{l}$. Eine Computertomographieaufnahme zeigte das Bild einer Pneumonie in moderater Ausprägung, suggestiv für eine SARS-CoV-2Pneumonie und ohne Nachweis einer Lungenarterienembolie. Die ophthalmo- 


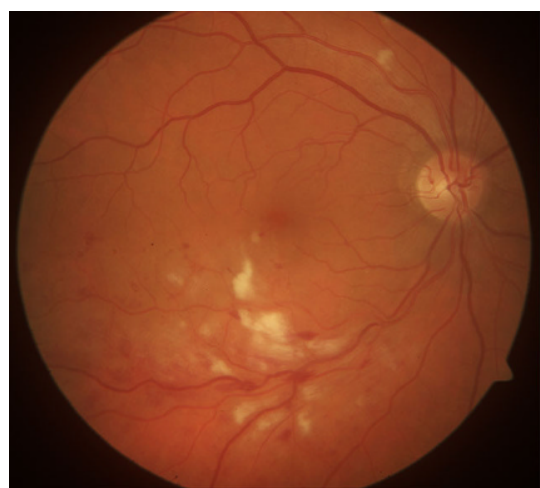

Abb. $1 \Delta$ Fundusfoto des rechten Auges mit moderater Venenstauung des temporal-inferioren Gefäßbogens. Begleitend zeigen sich gefäßassoziierte Streifenblutungen sowie CottonWool-Spots. Insgesamt weist das Fundusbild Aspekte eines venösen retinalen Verschlusses als auch einer CMV-Retinitis auf und erinnert somit an ein Pizza-ähnliches Aussehen

logische Untersuchung erbrachte den gleichen Befund wie 1 Woche zuvor. Der Bindehautabstrich war jedoch zu dem Zeitpunkt auf beiden Augen negativ für SARS-CoV-2-RNA.

Während des stationären Aufenthaltes verbesserte sich sein Befund, und er konnte nach 7 Tagen erneut mit einem guten Allgemeinbefinden entlassen werden.

Der Patient erhielt eine Anschlussbehandlung mit intravitrealen Antivasculargrowth-factor-Injektionen ins rechte $\mathrm{Au}$ ge (insgesamt bisher 4 Injektionen zum Zeitpunkt der Einreichung) sowie fokale Laserkoagulation der ischämischen Areale. Hierunter kam es zu einem Rückgang des zystoiden Makulaödems im OCT mit Visusanstieg auf 0,9.

\section{Diskussion}

Der Patient in unserem Fall berichtete ca. 10 bis 14 Tage vor dem Auftreten von generellen COVID-19-Symptomen über Sehstörungen auf dem rechten Auge. Klinisch zeigten sich Zeichen einer Venenastthrombose (VAT) mit ausgeprägten Cotton-WoolSpots, intraretinalen Streifenblutungen sowie Makulaödem. Insgesamt erinnert das pizzaähnliche Fundusbild auch an eine Zytomegalievirusretinitis. In der Fluoreszeinangiographie zeigte sich jedoch das Bild eines ischämischen Venenastverschlusses derinferioren Temporalvene. Auch die Min-

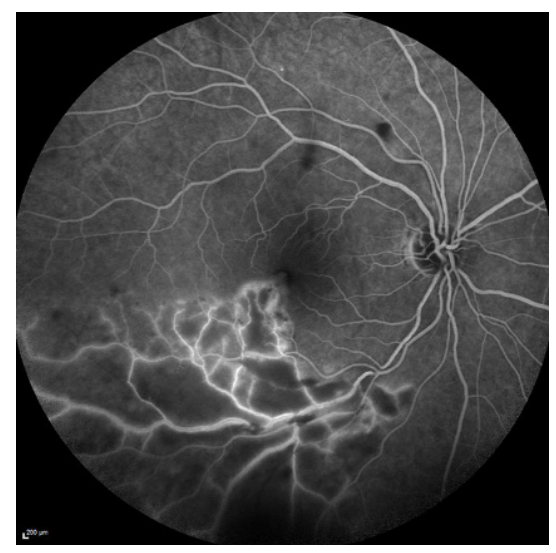

Abb. $2 \Delta$ Spätphase einer Fluoreszeinangiographie: Es zeigen sich am inferior-temporalen Gefäßbogen moderat gestaute venöse Gefäße mit eingeschlossener retinaler Ischämie sowie Endotheldysfunktion mit gefäßassoziierter $\mathrm{Hy}$ perfluoreszenz. Auch die Lokalisation der Okklusion an einer Gefäßkreuzungsstelle spricht eindeutig für eine VAT und schließt die Differenzialdiagnose einer CMV-Retinitis aus

derperfusion nach einer Gefäßkreuzungsstelle spricht eindeutig für einen Venenastverschluss. Eine virusassoziierte Retinitis oder retinale Nekrose ist nicht zu sehen. Wir können nicht mit Sicherheit unterscheiden, ob die VAT durch die COVID-19-Erkrankung ausgelöst wurde oder im Zusammenhang mit systemischen Komorbiditäten zu sehen ist. Es zeigte sich neben der VAT inferior auch ein Cotton-Wool-Spot am superioren Gefäßbogen (• Abb. 1). Dies ist für eine reine VAT eher untypisch und spricht für das Vorliegen gefäßassoziierter Komorbiditäten. Jedoch fanden sich in der kardiovaskulären Ursachenabklärung keine Risikofaktoren für das Auftreten eines thrombogenen Ereignisses. Ebenso gab es auf dem Partnerauge fundoskopisch bis auf eine einzelne Hämorrhagie unterhalb der Papille keine Zeichen eines chronischen arteriellen Hypertonus. Ein durchgeführtes Thrombophiliescreening blieb negativ. Wir vermuten daher eher eine Assoziation mit der COVID-19-Infektion, auch in Anbetracht der häufig berichteten vaskulären Nebenwirkungen bei COVID-19. Auch wenn der Pathomechanismus noch nicht vollständig geklärt ist, erhöhen hyperinflammatorische Reaktionen das Risiko für venöse thrombembolische Ereignisse [1]. In der SERPICO-Studie konnten retinale Gefäßaffektionen bei COVID-19Patienten nachgewiesen werden. Die Autoren fanden heraus, dass der venöse Ge- fäßdurchmesser bei Erkrankten signifikant größer war als bei gesunden Probanden. Des Weiteren schien der Gefäßdurchmesser positiv mit der Schwere der COVID19-Infektion zu korrelieren [2]. Vaskuläre Komplikationen sind auch nach COVID-19Impfungen beschrieben. Der impfstoffassoziierten Thrombozytopenie („vaccine-induced immune thrombotic thrombocytopenia" [VITT]) liegt wahrscheinlich eine Autoimmunreaktion auf COVID-19-Impfstoffe zugrunde, welche zu Thrombozytopenien und Thrombosen führen kann. Der genaue Pathomechanismus dieser seltenen Komplikation ist bisher unbekannt, ebenso wie die Bedeutung im Hinblick auf mögliche retinale Gefäßverschlüsse nach der Impfung [3, 4].

Interessanterweise zeigte sich außerdem an beiden Augen ein positiver Bindehautbefund auf SARS-CoV-2. Sowohl eine Ausbreitung per continuitatem als auch hämatogen oder neurogen in die Retina sind denkbar [5]. Auch konnte bereits SARS-CoV-2 in retinalem Gewebe (post mortem) nachgewiesen werden [6]. Retinale Manifestationen von COVID-19 sind folglich denkbar.

Unserer Erkenntnis nach, ist unser Fall der erste, welcher einen retinalen Venenastverschluss als Erstmanifestation einer COVID-19-Pneumonie beschreibt. Eine kürzlich publizierte Fallbeschreibung berichtete über einen 40-jährigen COVID-19-Patienten mit Pneumonie, tiefer Beinvenenthrombose sowie bilateralem Zentralvenenverschluss [7]. Auch gibt es eine Reihe weiterer Case Reports über retinale Verschlüsse bei jungen, gesunden COVID-19-Patienten [8, 9]. Berichte über retinale Thrombosen nach COVID19-Impfungen sind nach unserem Wissen jedoch bisher nicht veröffentlicht.

Mit dieser Kasuistik möchten wir dafür sensibilisieren, dass die Möglichkeit einer retinalen Venenthrombose als frühe hyperinflammatorische Reaktion einer COVID19-Erkrankung in Betracht gezogen werden sollte. Auch bestätigt unser Fall die aktuelle Empfehlung der Thrombose- und Embolieprophylaxe bei schwerer COVID19-Infektion [10]. 


\section{Fazit für die Praxis}

- Die aktuelle Studienlage zu retinalen Manifestationen bei COVID-19 ist sehr begrenzt. Bei vaskulären Pathologien des Fundus sollte auch an eine mögliche COVID-19-Erkrankung gedacht werden.

- SARS-CoV-2-RNA lässt sich in Tränenflüssigkeit nachweisen. Dies sollte als mögliche Infektionsquelle bedacht werden.

- Ophthalmologische Manifestationen könnten Frühsymptome einer schweren COVID-19-Pneumonie sein.

\section{Korrespondenzadresse}

\section{Dr. Kristin Hösel}

Universitätsklinikum Schleswig-Holstein Campus Kiel

Arnold-Heller-Str. 3, 24104 Kiel, Deutschland kristin.hoesel@uksh.de

Funding. Open Access funding enabled and organized by Projekt DEAL.

\section{Einhaltung ethischer Richtlinien}

Interessenkonflikt. K. Hösel, M. Saeger und J.B. Roider geben an, dass kein Interessenkonflikt besteht.

Für diesen Beitrag wurden von den Autoren keine Studien an Menschen oder Tieren durchgeführt. Für die aufgeführten Studien gelten die jeweils dort angegebenen ethischen Richtlinien. Für Bildmaterial oder anderweitige Angaben innerhalb des Manuskripts, über die Patienten zu identifizieren sind, liegt von innen und/oder ihren gesetzlichen Vertretern eine schriftliche Einwilligung vor.

Open Access. Dieser Artikel wird unter der Creative Commons Namensnennung 4.0 International Lizenz veröffentlicht, welche die Nutzung, Vervielfältigung, Bearbeitung, Verbreitung und Wiedergabe in jeglichem Medium und Format erlaubt, sofern Sie den/die ursprünglichen Autor(en) und die Quelle ordnungsgemäß nennen, einen Link zur Creative Commons Lizenz beifügen und angeben, ob Änderungen vorgenommen wurden.

Die in diesem Artikel enthaltenen Bilder und sonstiges Drittmaterial unterliegen ebenfalls der genannten Creative Commons Lizenz, sofern sich aus der Abbildungslegende nichts anderes ergibt. Sofern das betreffende Material nicht unter der genannten Creative Commons Lizenz steht und die betreffende Handlung nicht nach gesetzlichen Vorschriften erlaubt ist, ist für die oben aufgeführten Weiterverwendungen des Materials die Einwilligung des jeweiligen Rechteinhabers einzuholen.

Weitere Details zur Lizenz entnehmen Sie bitte der Lizenzinformation auf http://creativecommons.org/ licenses/by/4.0/deed.de.

\section{Literatur}

1. Helms J, Tacquard C, Severac F et al (2020) High risk of thrombosis in patients with severe SARSCoV-2 infection: a multicenter prospective cohort study. Intensive Care Med 46:1089-1098. https:// doi.org/10.1007/s00134-020-06062-x

2. Invernizzi A, Torre A, Parrulli $S$ et al (2020) Retinal findings in patients with COVID-19: results from the SERPICO-19 study. EClinicalMedicine 27:100550. https://doi.org/10.1016/j.eclinm.2020.100550

3. Aleem A, Nadeem AJ (2021) Coronavirus (COVID19) vaccine-induced immune thrombotic thrombocytopenia (VITT). http://www.ncbi.nlm.nih. gov/books/NBK570605/.Zugegriffen:5.Aug. 2021 (StatPearls)

4. Hunter PR (2021) Thrombosis after covid-19 vaccination. BMJ 373:n958. https://doi.org/10. 1136/bmj.n958

5. de Figueiredo CS, Raony Í, Giestal-de-Araujo E (2020) SARS-CoV-2 targeting the retina: hostvirus interaction and possible mechanisms of viral tropism. Ocul Immunol Inflamm 28:1301-1304. https://doi.org/10.1080/09273948.2020.1799037

6. Casagrande M, Fitzek A, Püschel K et al (2020) Detection of SARS-CoV- 2 in human retinal biopsies of deceased COVID-19 patients. Ocul Immunol Inflamm 28:721-725. https://doi.org/10.1080/ 09273948.2020.1770301

7. Gaba WH, Ahmed D, Nuaimi RKA et al (2020) Bilateral central retinal vein occlusion in a $40-$ year-old man with severe coronavirus disease 2019 (COVID-19) pneumonia. Am J Case Rep 21:e927691-1-e927691-5. https://doi.org/10. 12659/AJCR.927691

8. Finn AP, Khurana RN, Chang LK (2021) Hemi-retinal vein occlusion in a young patient with COVID-19. Am J Ophthalmol Case Rep 22:101046. https://doi. org/10.1016/j.ajoc.2021.101046

9. Yahalomi T, Pikkel J, Arnon R et al (2020) Central retinal vein occlusion in a young healthy COVID19 patient: a case report. Am J Ophthalmol Case Rep 20:100992. https://doi.org/10.1016/j.ajoc. 2020.100992

10. Bikdeli B, Madhavan MV, Jimenez D et al (2020) COVID-19 and thrombotic or thromboembolic disease: implications for prevention, antithrombotic therapy, and follow-up. J Am Coll Cardiol 75:2950-2973. https://doi.org/10.1016/j.jacc. 2020.04.031 